\title{
Relapsing granulomatosis with polyangiitis with severe lung and upper respiratory tract involvement successfully treated with rituximab
}

\author{
Anna Masiak, Zbigniew Zdrojewski \\ Department of Interial Diseases, Connective Tissue Diseases and Geriatrics, University of Gdansk, Gdansk, Poland
}

\begin{abstract}
Granulomatosis with polyangiitis (GPA) is a chronic, relapsing, primary systemic small vessel vasculitis associated with anti-neutrophil cytoplasmic antibodies. The disease is characterised by the involvement of various organs. The relapse rate rises from about $20 \%$ at 12 months to about $60 \%$ at 5 years. The combination of glucocorticoids and cyclophosphamide remains the standard therapy for patients with generalised GPA; nevertheless, some patients do not respond satisfactorily to this treatment. According to EULAR-EDTA recommendations for the management of ANCA-associated vasculitis, RTX should be considered for remission-induction of new-onset as well as major relapse of organ-threatening or life-threatening GPA. Here we present a 35-year-old patient with relapsing GPA successfully treated with RTX. We also highlight the infectious complications of immunosuppressive treatment.
\end{abstract}

Key words: granulomatosis with polyangiitis, rituximab, lung involvement, nasal carriage Stapylococcus aureus.

\section{Introduction}

Granulomatosis with polyangiitis (GPA) belongs to the group of primary systemic small and medium vessels vasculitis associated with the presence of anti-neutrophil cytoplasmic antibodies (ANCA-associated vasculitis AAV) [1]. This chronic relapsing disease is characterised by the involvement of various organs. The classic triad of symptoms includes the involvement of the upper and lower respiratory tract and kidneys. The disease can occur in several forms, from mild to very severe and life-threatening one. Relapse rate rises from about 20\% at 12 months to about $60 \%$ at 5 years [2]. Since the introduction of steroids and cyclophosphamide as a standard immunosuppressive regimen, survival of patients diagnosed with GPA has improved considerably. Mortality rates, previously exceeding $80 \%$ in 1 year, have been reduced to $20 \%$ in 5 years [3], but still it is a chronic disease, significantly lowering the quality of the patient's life. Rituximab (RTX) is a monoclonal anti-CD20 antibody that selectively targets B cells. According to
EULAR-EDTA recommendations for the management of ANCA-associated vasculitis RTX should be considered for remission-induction of new-onset as well as major relapse of organ-threatening or life-threatening AAV [4]. Here we present a patient with relapsing GPA successfully treated with RTX. We also highlight the problem of infectious complications of immunosuppressive treatment.

\section{Case report}

A 35-year-old woman was admitted to our department in August 2012 due to recurrent respiratory infections, nasal congestion and purulent nasal discharge without improvement after antibiotics. The patient also suffered from ulcerated subcutaneous nodules identified as pyoderma gangrenosum, fever, weakness, arthralgia and arthritis for 6 months before hospitalization. The physical examination revealed inflammatory granulomatous tissue with crusts in the nose, nasal septum perforation and skin ulceration on the leg. 
Laboratory tests were significant for normocytic normochromic anaemia (haemoglobin $10.2 \mathrm{~g} / \mathrm{dl}$ ), mild leucocytosis (white blood cells 11.3/l), thrombocytosis (PLT $437 \mathrm{G} / \mathrm{l}$ ), erythrocyte sedimentation rate (ESR) $42 \mathrm{~mm} / \mathrm{h}$ (normal range $2-0 \mathrm{~mm} / \mathrm{h}$ ) and C-reactive protein (CRP) $37 \mathrm{mg} / \mathrm{dl}$ (normal range $0-5$ ). Urine test and creatinine level were normal. Blood test revealed positivity for PR3ANCA antibodies (CANCA 1: 40, anti-PR3 41 RU/ml, positive $>20 \mathrm{RU} / \mathrm{ml}$ ). Nasal cultures were positive for Staphylococcus sp. Computed tomography (CT) of the chest showed bilateral solid nodules with cavitation (Fig. 1).

Based on clinical presentation with upper and lower respiratory tract involvement, typical lung changes on CT and presence of CANCA antibodies, the granulomatosis with polyangiitis (GPA) was recognized. Its' clinical activity was assessed using a disease-specific activity index the Birmingham Vasculitis Activity Score modification for GPA (BVAS/GPA = 5 points). The patient was treated with glucocorticoid pulses (methylprednisolone $3 \times 1,0$ gi.v.) and pulses of cyclophosphamide (CYC) in dose $15 \mathrm{mg} / \mathrm{kg} /$ infusion, according to EUVAS recommendations with frequency of administration: initial 3 pulses every 2 weeks, then every 3 weeks. Prophylaxis against infection with Pneumocystis jirovecii with trimethoprim/sulfamethoxazole was also administrated. After 6 months of treatment (total dose of CYC $7.0 \mathrm{~g}$ i.v.), skin changes have healed, but ANCA titers increased (CANCA 1 : 320) and control CT imaging revealed persistent pulmonary infiltrations (Fig. 2).

Considering the patient's age and the desire to have another child, it was decided to change the immunosup- pressive treatment to mycophenolate mofetil (MMF). Unfortunately, the control CT of the chest performed after the subsequent 3 months of MMF treatment revealed a significant progression of lung nodules (Fig. 3). CYC was administrated again to the total dose of $11.8 \mathrm{~g}$ i.v. The activity of the disease remained high (BVAS/GPA $=8$ points) with new clinical symptoms - strawberry gingivitis, mild polyneuropathy, progression of sinuses involvement with destruction of the maxillary sinus bone structures (Fig. 4) and orbital pseudotumor causing stare of the left eyeball (Fig. 5).

Laboratory test preformed that time showed anemia (Hgb $9.8 \mathrm{~g} / \mathrm{dl}$ ), thrombocytosis (PLT $425 \mathrm{G} / \mathrm{l})$ ), high CRP (149 $\mathrm{mg} / \mathrm{l})$. There were no laboratory symptoms of renal involvement (creatinine concentration and urine tests were normal). Nasal, blood and bronchoalveolar lavage cultures were negative. Due to resistance to cyclophosphamide, the rituximab (RTX) was administrated in total dose of $2.0 \mathrm{~g}$ i.v. $(2 \times 1.0$ g every 2 weeks). During assessment 6 months after RTX infusion, the clinical remission was noted (BVAS/ $\mathrm{GPA}=0$ points), the PR3-ANCA titres decreased significantly (anti-PR3 $11 \mathrm{RU} / \mathrm{ml}$ ), laboratory tests were within normal ranges and chest $C T$ revealed significant regression of the pulmonary infiltrations (Fig. 6).

For remission-maintenance 2.0 grams of RTX were administrated. Two months later the patient was hospitalised due to soft tissue swelling in the left half of the face covering the area around the nose and left eye causing limitation of vision in this eye. Moreover, ophthalmological examination revealed purulent infection of the lacrimal sack in left eye. In laboratory tests eleva-

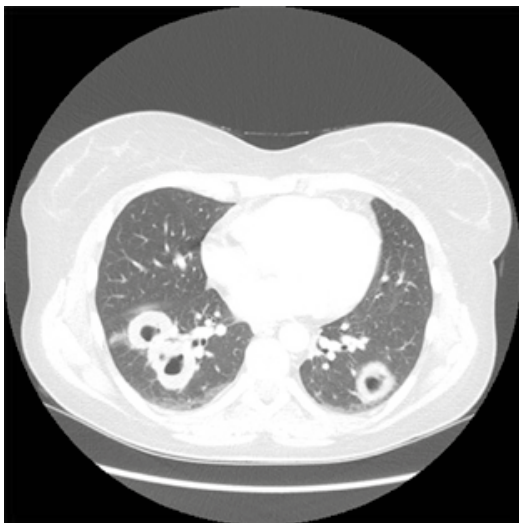

Fig. 1. Chest CT at diagnosis - bilateral solid nodules, cavitation with irregular, thickwalled cavity margins typical for GPA.

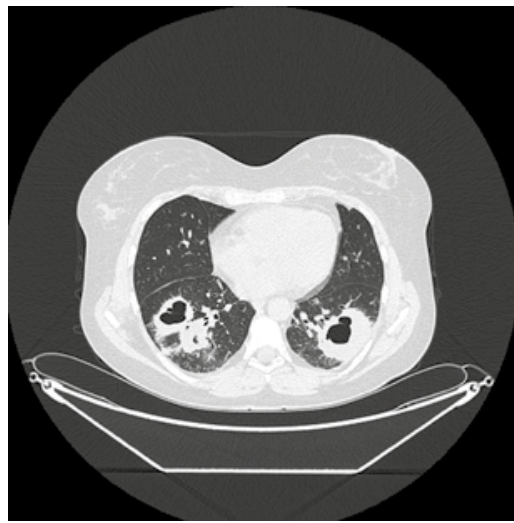

Fig. 2. Chest CT after 6 months of immunosuppressive treatment; in the lower lobes of both lungs infiltrates in the form of nodular mass with cavitations and regions of consolidation involving almost entirely both lower lobes, merging with each other and adjacent to the pleura.

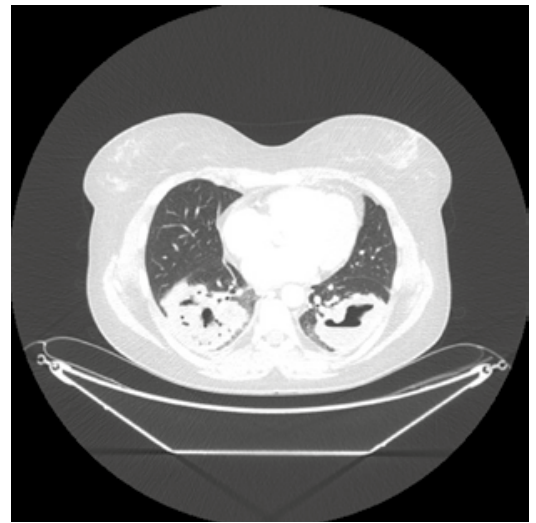

Fig. 3. Chest CT after another 3 months treatment with mycophenolate mofetil; further progression of consolidations in both lungs. 


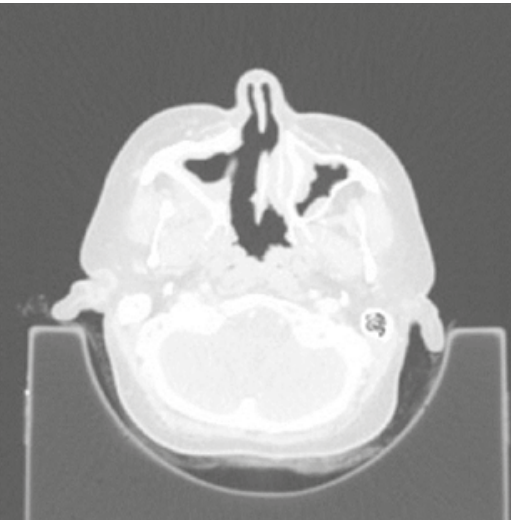

Fig. 4. Destruction of the maxillary sinus bone structures.

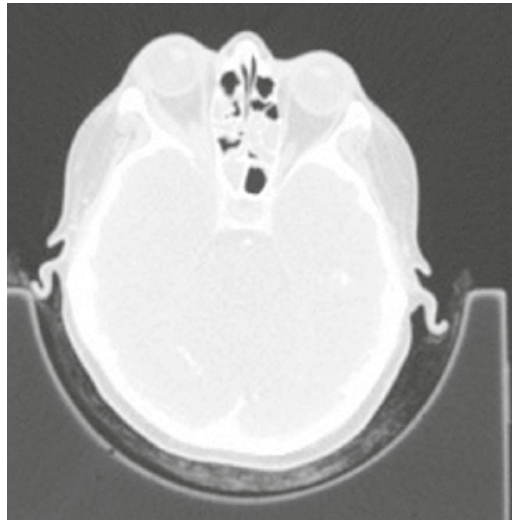

Fig. 5. Orbital pseudotumor causing stare of the left eyeball.

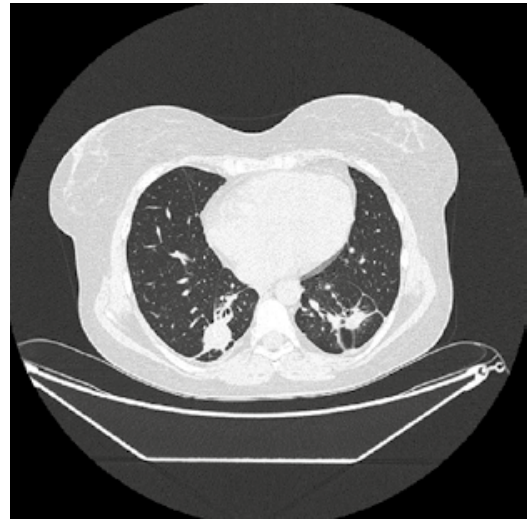

Fig. 6. CT of the chest after treatment with rituximab; regression of changes, no new infiltrates in the lungs. tion of acute inflammatory markers (CRP $42 \mathrm{mg} / \mathrm{l}$, ESR $32 \mathrm{~mm} / \mathrm{h}$ ) was observed. Cultures from lacrimal sac were positive for Pseudomonas aeruginosa. Antibiotic therapy and drainage of the lacrimal sack were administrated with good response. Four months later (6 months after second rituximab infusion) the activity of the disease was assessed, and patient was still in clinical and radiological remission (BVAS/GPA $=0$ points). Methotrexate (25 mg/week orally) was administrated for maintenance of remission. One year later (in May 2016) the patient started to complain about purulent nasal discharge and fever. Elevated inflammatory markers (CRP 60 mg/l, ESR $56 \mathrm{~mm} / \mathrm{h}$ ), mild anaemia (Hgb $10.6 \mathrm{~g} / \mathrm{l}$ ) and increased level of PR3-ANCA antibodies (CANCA 1 : 160, PR3 29 RU/ $\mathrm{ml}$ ) were observed in laboratory tests. Nasal cultures were positive for both Pseudomonas aeruginosa and Staphylococcus aureus. Radiological studies have shown a new lung infiltration and significant progression of destructive lesions in the bones of the head (Fig. 7).

Granulomatosis with polyangiitis relapse was recognised (BVAS/GPA $=2$ points). Due to previous resistance to CYC and good response to RTX, it was decided to re-infuse this biologic drug in a schedule different than the one used before $\left(375 \mathrm{mg} / \mathrm{m}^{2}\right.$ of body surface area, once a week for the next four infusions). After the third of four infusions, the purulent inflammation of the lacrimal sac reappeared. Cultures from the lacrimal sac showed Staphylococcus aureus infection. Antibiotic therapy was administrated for 14 days (amoxicillin with clavulanic acid) and a fourth infusion of RTX was given without further complications. After six months, the patient was in clinical remission, but several purulent infections of the lacrimal sac reappeared during that time. The patient was treated with methotrexate (25 mg/week) and small doses of glucocorticoids to maintenance the remission. Prophylaxis

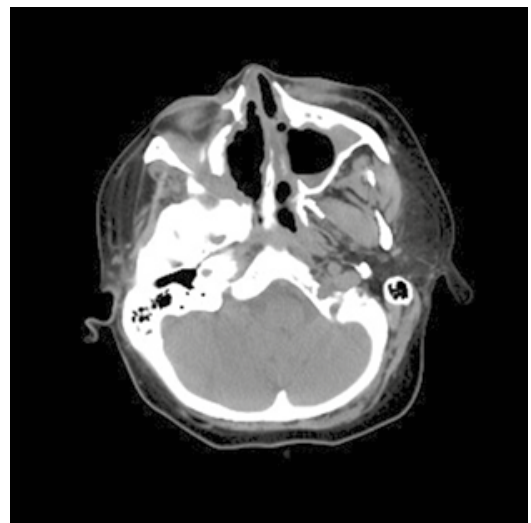

Fig. 7. CT of the head: significant progression of destructive lesions in the bones of the head.

against Pneumocystis jirovecii infection with trimethoprim/sulfamethoxazole was continued. Nasal cultures were repeated in case of any symptoms of infection and if results were positive for Staphylococcus aureus or Pseudomonas aeruginosa, antibiotic therapy was administrated.

\section{Discussion}

Granulomatosis with polyangiitis is a chronic relapsing disease. Cyclophosphamide and glucocorticoids have been the standard of remission induction therapy for generalized GPA for many years. CYC induction regimens are effective in $70-90 \%$ of patients, but a considerable number of patients are resistant to standard treatment. CYC exposure may also be associated with adverse effects such as infections, cancer, and infertility. The risk of ovarian failure depends essentially on the age at CYC 
initiation [5]. This aspect can be particularly important in middle-aged female patients, as in our case.

Rituximab is a chimeric monoclonal antibody directed against CD20, a cell surface antigen expressed almost exclusively on cells of B-lymphocyte lineage. Binding of the antibody to CD20 results in selective depletion of B lymphocytes by a variety of different mechanisms [6]. Rituximab has been used in AAV for induction of remission as in the RAVE [7] and RITUXVAS [8] trials, and in the maintenance as in the MAINRITSAN trial [9].

Observational studies investigating the efficacy of RTX in refractory GPA have been published in the recent years, most of which reported high rates of remission (80\%), even among patients with refractory disease and predominantly necrotising granulomatous disease manifestations [10-12]. Its use also allows a reduction of the exposure to cyclophosphamide. Rituximab may also be better than cyclophosphamide for those participants who are PR3-ANCA positive. Considering all new data, in 2016, the European League Against Rheumatism (EULAR) with the European Renal Association - European Dialysis and Transplant Association (ERA-EDTA) have published an update of 2009 EULAR recommendations with the focus on the management of ANCA-associated vasculitis [4]. The treatment differed according to clinical presentation of the disease. For remission-induction of new-onset organ-threatening or life-threatening AAV, treatment with a combination of glucocorticoids and either cyclophosphamide or rituximab is recommended. For patients with AAV refractory to remission-induction therapy, switching from cyclophosphamide to rituximab or from rituximab to cyclophosphamide is recommended. Nowadays, patients who have not responded to CYC have the option of treatment with RTX within the treatment program in Poland. This provides much better access to modern treatment. The possibility of such treatment from the beginning, would prevent our patient from prolonged exposure to CYC and its complications (ovarian failure).

The presented patient had severe upper respiratory tract involvement. It is worth highlighting the differences in recommendations regarding to this aspect. The 2009 recommendations suggested that methotrexate use was reasonable for those patients. However, this time the recommendations have differentiated even localised disease into that with and without cartilage and bony involvement, the argument being that destruction of nasal tissues is an organ threatening manifestation and the treatment is as above $[4,13]$. Mycophenolate mofetil was completely ineffective in our patient. According to new recommendations, MMF could be used in the niche area of localised disease where methotrexate might not be appropriate. Most patients should therefore receive either cyclophosphamide or rituximab for remission induction.

The risk of serious bacterial infectious after an immunosuppressive therapy, especially used in severe and refractory cases of systemic autoimmune diseases, is well known. There is a strong possibility that the initial adverse effects of remission induction are related to glucocorticoid treatment, as shown by the similar rate of adverse events in a randomised controlled trial of rituximab vs. cyclophosphamide [7]. Peripheral B cell depletion caused by rituximab lasts for an average of 6-9 months and sometimes longer. The risk of serious infectious events remains several months after an RTX course, primarily within 6 months of the RTX infusion [14]. Our patient suffered because of recurrent upper respiratory tract infections. Interestingly in our patient the main infectious complication was purulent lacrimal sac inflammation. The described patient did not have any of the identified risk factors associated with an increased risk of infection in patients treated with RTX: corticosteroids were used mainly in low doses ( $<15 \mathrm{mg} /$ day), she did not have kidney failure or diabetes, her lgG level was normal, and she was treated with methotrexate but not with azathioprine $[15,16]$. Taking that into consideration, it can be assumed that frequent recurrences may have been associated with chronic nasal carriage of Staphylococcus sp. It was shown, that GPA might be precipitated by infection with Staphylococcus aureus, which initiates a cascade of immunological events caused by several Staphylococcus aureus toxins. This hypothesis is supported by the observation that chronic nasal carriage of Staphylococcus aureus is related to exacerbations of GPA [17]. However, some researchers report that the transmission of Staphylococcus aureus to RTX-treated GPA patients did not affect GPA exacerbations or treatment efficacy and even reduced the risk of significant hypogammaglobulinaemia in these patients [18]. So, the topic is open, but patients' carriers of bacterial infections including Staphylococcus aureus need special attention.

\section{Conclusions}

The combination of glucocorticoids and cyclophosphamide remains the standard therapy for patients with generalised GPA. Nevertheless, ANCA-associated vasculitis (AAV) has a high relapse rate, and some patients do not respond satisfactorily to this treatment. Many results indicate that rituximab therapy has a valuable effect in patients with AAV and may be superior to CYC in relapsing disease. Rituximab as an effective and well-tolerated treatment for patients with AAV should be strongly considered in severely affected patients who 
do not respond to standard therapy. Staphylococcus au reus carriers in patients with autoimmune diseases, often hospitalized and treated with immunosuppressants should be considered as a potential cause of exacerbation of a disease [19].

The authors declare no conflict of interest.

\section{References}

1. Jennette JC. Nomenclature and classification of vasculitis: lessons learned from granulomatosis with polyangiitis. Clin Exp Immunol 2011; 164 (Suppl 1): 7-10.

2. Mukhtyar C, Luqmani R. Diseasespecific quality indicators, guidelines, and outcome measures in vasculitis. Clin Exp Rheumatol 2007; 25 (6 Suppl 47): 120-129.

3. Bligny D, Mahr A, Toumelin P, et al. Predicting mortality in systemic Wegener's granulomatosis; a survival analysis based on 93 patients. Arthritis Rheum 2004; 51: 83-91.

4. Yates $M$, Watts RA, Bajemna IM, et al. EULAR-EDTA recommendations for the management of ANCA-associated vasculitis. Ann Rheum Dis 2016; 10: 1-12.

5. Huong DL, Amoura Z, Duhaut P, et al. Risk of ovarian failure and fertility after intravenous cyclophosphamide. A study in 84 patients. J Rheumatol 2002; 29: 2571-2576.

6. Weiner GJ. Rituximab: mechanism of action. Semin Hematol 2010; 47: 115-123.

7. Stone JH, Merkel PA, Spiera R, et al. Rituximab versus cyclophosphamide for ANCA-associated vasculitis. N Engl J Med 2010; 363: 221-232.

8. Jones RB, Tervaert JW, Hauser T, et al. Rituximab versus cyclophosphamide in ANCA-associated renal vasculitis. N Engl J Med 2010; 363: 211-220.

9. Guillevin L, Pagnoux C, Karras A, et al. Rituximab versus azathioprine for maintenance in ANCA-associated vasculitis. N Engl J Med 2014; 371: 1771-1780.

10. Keogh KA, Ytterberg SR, Fervenza FC, et al. Rituximab for Refractory Wegener's Granulomatosis Report of a Prospective, Open-Label Pilot Trial. Am J Respir Crit Care Med 2006; 173; 180-187.

11. Cartin-Ceba R, Golbin JM, Keogh KA, et al. Rituximab for remission induction and maintenance in refractory granulomatosis with polyangiitis (Wegener's): ten-year experience at a single center. Arthritis Rheum 2012; 64: 3770-3778.

12. Seo P, Specks U, Keogh KA. Efficacy of rituximab in limited Wegener's granulomatosis with refractory granulomatous manifestations. J Rheumatol 2008; 35: 2017-2023.

13. Sznajd J, Mukhtyar C. How to treat ANCAassociated vasculitis: practical messages from 2016 EULAR/ERAEDTA recommendations. Pol Arch Med Wewn 2016; 126: 781- 788.

14. Tony HP, Burmester G, Schulze-Koops H, et al. Safety and clinical outcomes of rituximab therapy in patients with different autoimmune diseases: experience from a national registry (GRAID). Arthritis Res Ther 2011; 13: R75.

15. Heusele M, Clerson P, Guery B, et al. Risk factors for severe bacterial infections in patients with systemic autoimmune diseases receiving rituximab. Clin Rheumatol 2014; 33: 799-805.
16. Trivin C, Tran A, Moulin B, et al. Infectious complications of a rituximab-based immunosuppressive regimen in patients with glomerular disease. Clin Kid J 2017; 10: 461-469.

17. Popa ER, Stegeman CA, Kallenberg CG, Tervaert JW. Staphylococcus aureus and Wegener's granulomatosis. Arthritis Res 2002; 4: 77-79.

18. Besada E, Koldingsnes W, Nossent JC. Staphylococcus Aureus carriage and long-term Rituximab treatment for Granulomatosis with polyangiitis. Peer J 2015; 3: e1051.

19. Varley CD, Deodhar AA, Ehst BD, et al. Persistence of Staphylococcus aureus colonization among individuals with immune-mediated inflammatory diseases treated with TNF- $\alpha$ inhibitor therapy. Rheumatology (Oxford) 2014; 53: 332-337. 